\title{
Diode like high-contrast asymmetric transmission of linearly polarized waves based on plasmon-tunneling effect coupling to electromagnetic radiation modes
}

\author{
Bahram Khalichi $^{1,2, *}$ (D) Amir Ghobadi ${ }^{1,2}$, Ataollah Kalantari Osgouei ${ }^{1,3}$ (D) \\ and Ekmel Ozbay ${ }^{1,2,3,4, *}$ \\ 1 NANOTAM-Nanotechnology Research Center, Bilkent University, 06800 Ankara, Turkey \\ 2 Department of Electrical and Electronics Engineering, Bilkent University, 06800 Ankara, Turkey \\ 3 Department of Physics, Bilkent University, 06800 Ankara, Turkey \\ ${ }^{4}$ UNAM-Institute of Materials Science and Nanotechnology, Bilkent University, Ankara, Turkey \\ E-mail: bahram@ee.bilkent.edu.tr and ozbay@bilkent.edu.tr
}

Received 29 March 2021, revised 25 May 2021

Accepted for publication 11 June 2021

Published 25 June 2021

\begin{abstract}
We present a narrow-band optical diode with a high-contrast forward-to-backward ratio at the near-infrared region. The design has a forward transmission of approximately $88 \%$, and a backward one of less than $3 \%$, yielding a contrast ratio of greater than $14.5 \mathrm{~dB}$ at a wavelength of $1550 \mathrm{~nm}$. The structure is composed of a one-dimensional diffraction grating on top of a dielectric slab waveguide, both of which are made of silicon nitride $\left(\mathrm{Si}_{3} \mathrm{~N}_{4}\right)$, and all together are placed over a silver (Ag) thin film embedded on a dielectric substrate. Utilizing a dielectric-based diffraction grating waveguide on a thin silver layer leads to the simultaneous excitation of two surface plasmon modes known as long- and short-range surface plasmon polaritons (SPPs) at both interfaces of the metallic layer. The plasmon-tunneling effect, which is the result of the coupling of SPPs excited at the upper interface of the metallic layer to the radiation modes, provides a high asymmetric transmission (AT) property. The spectral response of the proposed high-contrast AT device is verified using both rigorous coupled-wave analysis as an analytical approach and finite difference time domain as a numerical one.
\end{abstract}

Keywords: asymmetric transmission, diffraction grating, surface plasmon polaritons, plasmon tunneling

(Some figures may appear in colour only in the online journal)

\section{Introduction}

Allowing the light transmission from one direction, while suppressing transmission from the opposite side is one of the popular and significant phenomena realized in various optical

\footnotetext{
* Authors to whom any correspondence should be addressed.
}

applications [1-9]. This nonreciprocal optical-diode behavior $[8,9]$ is utilized to control one-way light transmission, increase the efficiency, and reduce the damage in the optical communication circuitry, such as isolators, rectifiers, logic circuits, and clippers. As a common approach, symmetry-broken chiral metamaterials along with multilayer design and sophisticated structures are utilized to achieve customized and simultaneous functionalities, such as polarization-sensitive asymmetric 
transmission (AT) $[10,11]$. This AT phenomenon originates from the interaction of electromagnetic radiation with the structural two-dimensional chirality in the metamaterials $[12,13]$. As a result, a portion of an incident electromagnetic wave is converted to its cross-polarized counterpart for both forward and backward illuminations, while the unidirectional transmission (optical-diode like behavior) is not the goal in chiral metamaterial-based structures. In addition, the use of multilayer metallic configurations prevents obtaining a highcontrast AT ratio. Although optical devices with an AT characteristic can be realized using plasmonic metamaterials as another approach [5-7], they usually exhibit low transmission amplitudes due to optical losses in the multilayer designs [14-20]. Moreover, the realization of a double grating design separated by a thin dielectric is a challenging task from the fabrication perspective [21-23]. Therefore, in an ideal scheme, it is required to reduce the optical loss (thinning the metallic components down to sub-wavelength geometries) while keeping the overall design simple. The excitation of surface plasmon polaritons (SPPs) over both boundaries of a thin metal slab is widely utilized in biochemical sensing applications [24-26], and it can be an innovative approach to be exploited in diode-like optical devices. Furthermore, based on previous reports, the outcoupling of SPP modes to radiation modes [2732] with an efficiency of $50 \%$ is proposed in [27]. In addition, using a modified structure, the transmittance of $68 \%$ is reported in [28] and the enhancement of the transmittance up to $72 \%$ is predicted in [29]. Therefore, adopting this design architecture for an optical diode application could provide the opportunity to achieve high-contrast AT for narrow-band applications in the near infrared (NIR) region.

In the present paper, we demonstrate a high-contrast and a narrowband optical diode that operates based on the plasmontunneling effect [21, 33, 34]. The proposed optical diode is made of the dielectric grating waveguide over a thin silver (Ag) layer embedded on a thick substrate. This simple geometry is significantly distinct from other available plasmonicmetamaterial-based AT devices with multilayer configurations [35], multilayer metasurfaces [36], or subwavelength slits [23]. When the proposed device is illuminated by a forward incident light, the symmetric and antisymmetric plasmonic modes are excited by the diffraction grating waveguide at the bottom and top interfaces of the thin Ag interlayer, respectively. The antisymmetric mode is coupled to radiation modes in the next environment (substrate), while the symmetric mode is absorbed inside the metallic interlayer. Since the antisymmetric SPP mode is excited on the top interface of the metallic interlayer and outcoupled to radiation modes in the next environment, a high forward transmission can be achieved based on the plasmon-tunneling effect. Contrarily, under the backward illumination, the device offers a near-zero backward transmission thanks to the strong reflection of the Ag layer.

\section{Design methodology, operation principle, and simulation results}

A schematic diagram of the proposed structure with AT property, using the generation and outcoupling of SPPs, is presented in figure 1(a). The structure consists of a dielectric diffraction grating as a one-dimensional array on top of a same-material dielectric slab waveguide and a thin metal film, deposited onto a dielectric substrate. In figure 1(a), $t_{\mathrm{g}}$, $w_{\mathrm{g}}$, and $\Lambda_{3} \mathrm{~N}_{4}$ ) with the permittivity of 4.2025 , where the thickness of the dielectric slab waveguide is represented by $t_{\mathrm{d}}$. The dielectric slab forms a Fabry-Pérot cavity, which is used to improve the coupling efficiency. The thickness of the dielectric slab will also give us another degree of freedom on the spectral tuning of the resonance wavelengths [37]. Then, a thin Ag layer with the thickness of $t_{\mathrm{m}}$ is considered between the dielectric slab waveguide and the substrate (silicon dioxide: $\mathrm{SiO}_{2}$ ). The spectral refractive index of $\mathrm{Ag}$ is taken from the Johnson and Christy database [38]. Conceptually, when the proposed structure is normally illuminated by an $x$-polarized (TM-polarized wave: electric field is parallel and magnetic field is perpendicular to the plane of incidence [39]) uniform plane wave, the incident wave is diffracted by the grating, and then coupled into the waveguide and SPP modes at certain resonance wavelengths (in which the guided-mode condition of the planar waveguide and the phase-matched conditions are satisfied simultaneously). These SPPs are modulated by the grating and can be outcoupled to the radiation modes in the substrate [29]. Therefore, the grating is not only responsible for the coupling of the incident light to the SPPs, but also for outcoupling the resonant SPPs to the radiation modes. To numerically analyze this structure, the eigen-solver (to find the eigenvalues (resonance wavelengths of the entire structure) and the eigenvectors (the corresponding field distributions)) is exploited to optimize the structure to have an AT at a wavelength of approximately $1550 \mathrm{~nm}$. Accordingly, as shown in figure 1(a), the period and the width of the dielectric grating are set to be $\Lambda=1100 \mathrm{~nm}$ and $w_{\mathrm{g}}=880 \mathrm{~nm}$, respectively, which leads the filling factor equal to 0.8 . The thickness (height) of the grating $\left(t_{\mathrm{g}}\right)$ and the dielectric slab $\left(t_{\mathrm{d}}\right)$ are $260 \mathrm{~nm}$ and $470 \mathrm{~nm}$, and $t_{\mathrm{m}}$, the thickness of the metal film, is chosen as $20 \mathrm{~nm}$ to allow the excitation of both long- and short-range SPPs (LRSPPs and SRSPPs) [40, 41]. These parameters determine the resonance wavelength location in the NIR region at approximately $1550 \mathrm{~nm}$. Next, we computed the reflection, transmission spectra using the rigorous coupled-wave analysis (RCWA) as an analytical approach [42] besides the finite difference time domain (FDTD) software package as a numerical one. The calculated transmission and the reflection spectra when the structure is illuminated by an $x$-polarized uniform plane-wave propagating along the $\pm z$ directions (forward $(-z)$ and backward $(+z)$ illuminations) are given in figure 1(b). The obtained results from both methods are in good agreement with each other which demonstrate the accuracy of the simulations. As seen in this panel, the proposed structure exhibits an optical diode characteristic at a wavelength range of 1535-1563 nm (full width at half maximum: FWHM $/ \lambda_{0}=2 \%$ ). At $1549 \mathrm{~nm}$, the maximum power transmission of 0.88 occurs for the forward illumination, while the backward transmission value stayed below 0.03 . Therefore, the highest contrast ratio in the peak position is achieved as $14.67 \mathrm{~dB}$ (a ratio of about 29). To clearly scrutinize the origin of this distinct optical-diode 
behavior, we have to find out the nature of these resonance wavelengths.

To begin with, we simplified the geometry by the explanation of the conditions for the excitation of SPP modes in an insulator-metal-insulator (IMI) design. The configuration is presented in figure 1(c), and a thin metal film with the thickness of $t$ is considered. This configuration can support SPPs at both interfaces, where the excitation and overlapping of SPPs at both boundaries give rise to mixed modes, called symmetric and antisymmetric SPPs [40, 41]. The modes can be found by solving Maxwell's equations in each medium, and by satisfying the boundary conditions at both interfaces of the metallic layer. As the thickness of the metal slab decreases, the two modes become more distinct, where the symmetric SPP mode exhibits a less attenuation constant and effective refractive index, while the attenuation and effective refractive index of the antisymmetric one increase [24]. Therefore, according to the attenuation and propagation lengths, the symmetric mode is referred to as a LRSPP, while the antisymmetric mode is referred to as a SRSPP [40, 41]. For the analysis, a two-dimensional problem is considered (the width of the structure in the $y$ direction is infinite so that $\partial / \partial y=0)$. The geometry can support $\mathrm{TM}^{x}$ mode $\left(E_{x}, E_{z}\right.$ and, $H_{y}$ are the nonzero components). In this scenario, an $x$ directed magnetic vector potential $\left(A_{x}\right)$ can be represented as [39]:

$$
A_{x}(x, z)=\left\{\begin{array}{cc}
B_{1} \exp \left(-\alpha_{z}^{d_{1}} z-i k_{x} x\right) & z \geq \frac{t}{2} \\
{\left[A_{1} \cos \left(k_{z}^{m} z\right)+A_{2} \sin \left(k_{z}^{m} z\right)\right] \exp \left(-i k_{x} x\right)} & -\frac{t}{2} \leq z \leq \frac{t}{2} \\
B_{2} \exp \left(+\alpha_{z}^{d_{2}} z-i k_{x} x\right) & z \leq-\frac{t}{2}
\end{array}\right.
$$

where $\alpha_{z}^{d_{j}}(j=1,2)$ is the attenuation constant in the dielectric media, $k_{z}^{m}$ is the phase constants inside the metal layer, $k_{x}=k_{\mathrm{L}(\mathrm{S}) \mathrm{RSPP}}$ is the magnitude of the SPPs wavevector, and $A_{j}, B_{j}(j=1,2)$ are the unknown coefficients. The corresponding electric and magnetic fields, inside the metal and dielectric regions, can be calculated by:

$$
\begin{aligned}
E_{x}(x, z) & =\frac{-i}{\omega \mu_{0} \varepsilon_{j}}\left(\frac{\partial^{2}}{\partial x^{2}}+k_{x}^{2}\right) A_{x}(x, z) \\
& =\left\{\begin{array}{cc}
\frac{-i B_{1}}{\omega \mu_{0} \varepsilon_{d_{1}}}\left(-k_{x}^{2}+k_{d_{1}}^{2}\right) \exp \left(-\alpha_{z}^{d_{1}} z-i k_{x} x\right) & z \geq \frac{t}{2} \\
\frac{-i}{\omega \mu_{0} \varepsilon_{m}}\left(-k_{x}^{2}+k_{m}^{2}\right)\left[A_{1} \cos \left(k_{z}^{m} z\right)+A_{2} \sin \left(k_{z}^{m} z\right)\right] \exp \left(-i k_{x} x\right) & -\frac{t}{2} \leq z \leq \frac{t}{2} \\
\frac{-i B_{2}}{\omega \mu_{0} \varepsilon_{d_{2}}}\left(-k_{x}^{2}+k_{d_{2}}^{2}\right) \exp \left(+\alpha_{z}^{d_{2}} z-i k_{x} x\right) & z \leq-\frac{t}{2}
\end{array}\right.
\end{aligned}
$$

and

$$
\begin{aligned}
H_{y}(x, z) & =\frac{1}{\mu_{0}} \frac{\partial}{\partial z} A_{x}(x, z) \\
& =\left\{\begin{array}{cc}
\frac{B_{1}}{\mu_{0}}\left(-\alpha_{z}^{d_{1}}\right) \exp \left(-\alpha_{z}^{d_{1}} z-i k_{x} x\right) & z \geq \frac{t}{2} \\
\frac{1}{\mu_{0}}\left[-A_{1} k_{z}^{m} \sin \left(k_{z}^{m} z\right)+A_{2} k_{z}^{m} \cos \left(k_{z}^{m} z\right)\right] \exp \left(-i k_{x} x\right) & -\frac{t}{2} \leq z \leq \frac{t}{2} \\
\frac{B_{2}}{\mu_{0}}\left(+\alpha_{z}^{d_{2}}\right) \exp \left(+\alpha_{z}^{d_{2}} z-i k_{x} x\right) & z \leq-\frac{t}{2},
\end{array}\right.
\end{aligned}
$$


(a)
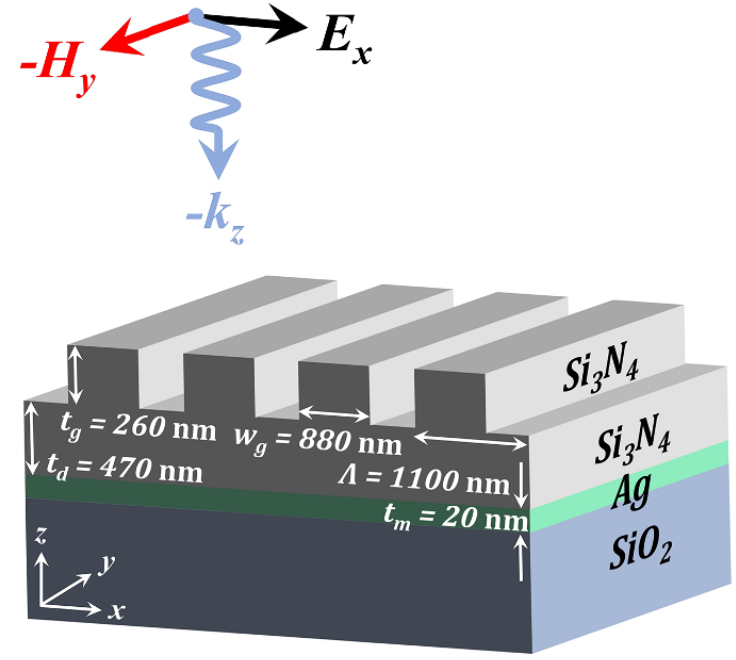

(b)
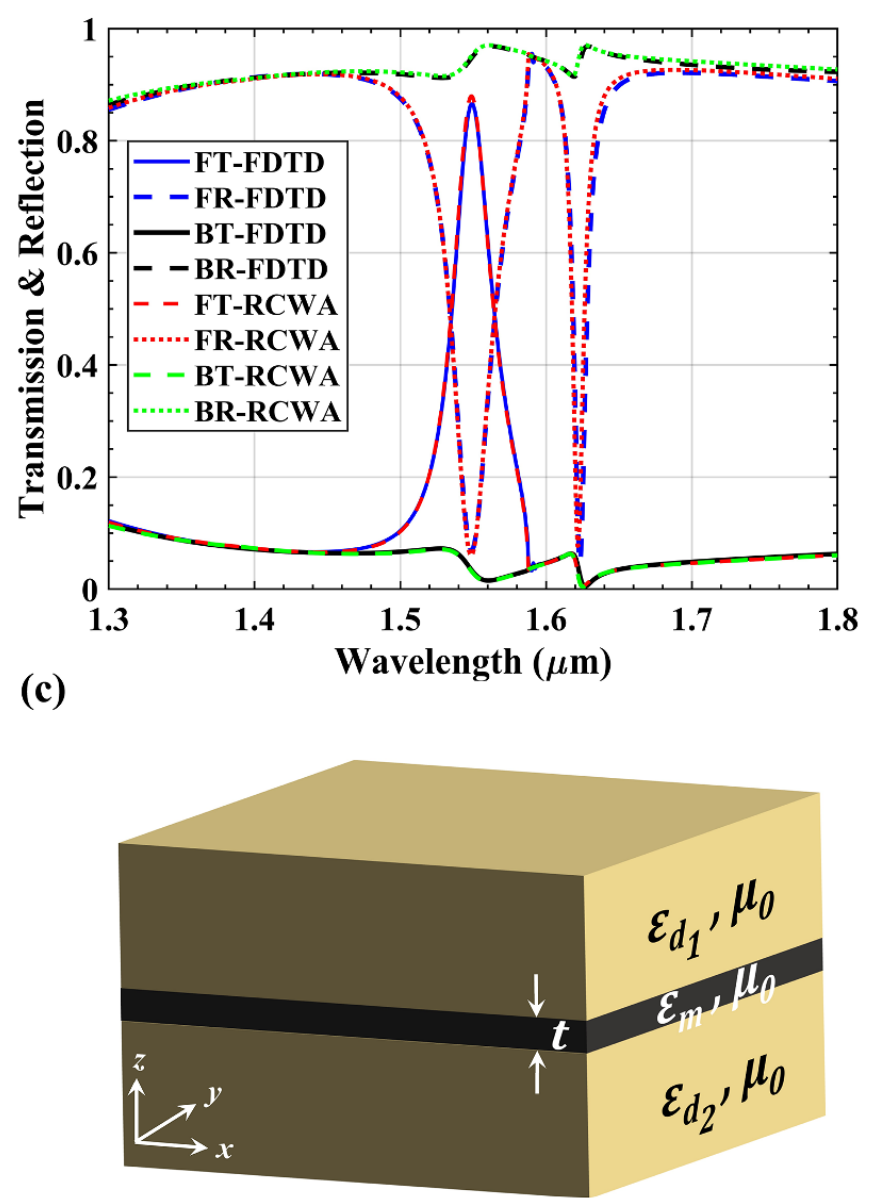

Figure 1. (a) Schematic diagram of the proposed optical-diode structure with a high AT characteristic designed based on dielectric diffraction grating waveguide on top of a thin metal film. The structure is normally illuminated by an $x$-polarized uniform plane wave from both sides. (b) Forward/backward transmission (FT/BT) and reflection (FR/BR) spectra of the proposed structure when the object is illuminated by an $x$-polarized uniform plane wave propagating along the $\pm z$ directions. (c) Schematic representation of a thin metal film with the thickness of $t$ surrounded by infinite dielectric media. respectively. Decomposing the electric- or magnetic-field distributions in the metal layer as a combination of symmetric and antisymmetric modes and satisfying the tangential boundary conditions at the interfaces, different dispersion relations $[40,41]$ for the SRSPP (antisymmetric) and LRSPP (symmetric) modes are obtained as:

$$
\begin{aligned}
& \text { SRSPP : } \quad \varepsilon_{m} \alpha_{z}^{d_{1}}+\varepsilon_{d_{1}} k_{z}^{m} \cot \left(k_{z}^{m} \frac{t}{2}\right)=0 \\
& \text { LRSPP : } \quad \varepsilon_{m} \alpha_{z}^{d_{2}}-\varepsilon_{d_{2}} k_{z}^{m} \tan \left(k_{z}^{m} \frac{t}{2}\right)=0,
\end{aligned}
$$

where $-\left(\alpha_{z}^{d_{j}}\right)^{2}=\varepsilon_{d_{j}} k_{0}^{2}-k_{\mathrm{L}(\mathrm{S}) \mathrm{RSPP}}^{2}, \quad$ and $\quad\left(k_{z}^{m}\right)^{2}=\varepsilon_{m} k_{0}^{2}-$ $k_{\mathrm{L}(\mathrm{S}) \mathrm{RSPP}}^{2}$. It should be noted here that the symmetric configuration of the transverse component $E_{z}$ or $H_{y}$ corresponds to the antisymmetric one for the longitudinal components $E_{x}$ and vice versa. In addition, the $z$ variation in the metal layer can be represented using hyperbolic functions that leads to some modifications in the final results. Based on (4a) and (4b), it can be concluded that SPs are excited on the top and bottom interfaces of the metal layer because of overlapping of the modes. Therefore, if the thickness of the metal film increases, the propagation constants of these two modes get closer, thereby leading to SPs on the top interface.

After a brief discussion regarding the possible excitation of different types of SPPs in IMI configuration, the nature of the resonance of the proposed optical-diode structure at the resonance wavelengths of 1549 and $1624 \mathrm{~nm}$ can be investigated. To achieve this purpose, five subsections are considered.

\subsection{Types of resonances}

Regarding the types of SPPs, the formation of two dip resonances in the reflection spectrum belonging to the forward illumination are due to the symmetric and antisymmetric SP mode excitations that result in absorption and transmission in the structure, respectively. The types and behaviors of the mentioned dip resonances in the reflection spectrum can be illustrated by utilizing dispersion relations given in (4a) and (4b). Accordingly, the SPPs should be dependent on different waveguide slab (superstrate) and substrate materials. Thereby, different materials are utilized as a substrate in the following order for the average refractive indices $\mathrm{CaF}_{2}<\mathrm{SiO}_{2}<\mathrm{BaF}_{2}<\mathrm{Al}_{2} \mathrm{O}_{3}$ (correspond to: $1.42<1.44<$ $1.46<1.74)$. In figure $2(\mathrm{a})$, it is evident that, as the average refractive index of the substrate increases, there is a red shift in the position of resonances from shorter toward longer wavelengths. However, a negligible shift in the position of the 1st resonance compared to the 2nd resonance is observed for different substrate materials. Clearly, the spectral separation between the modes increases by increasing the refractive index of the substrate. Based on the dispersion relations given in (4a) and (4b), SRSPP and LRSPP are dependent on superstrate and substrate materials, respectively. Although both SRSPP 
(a)
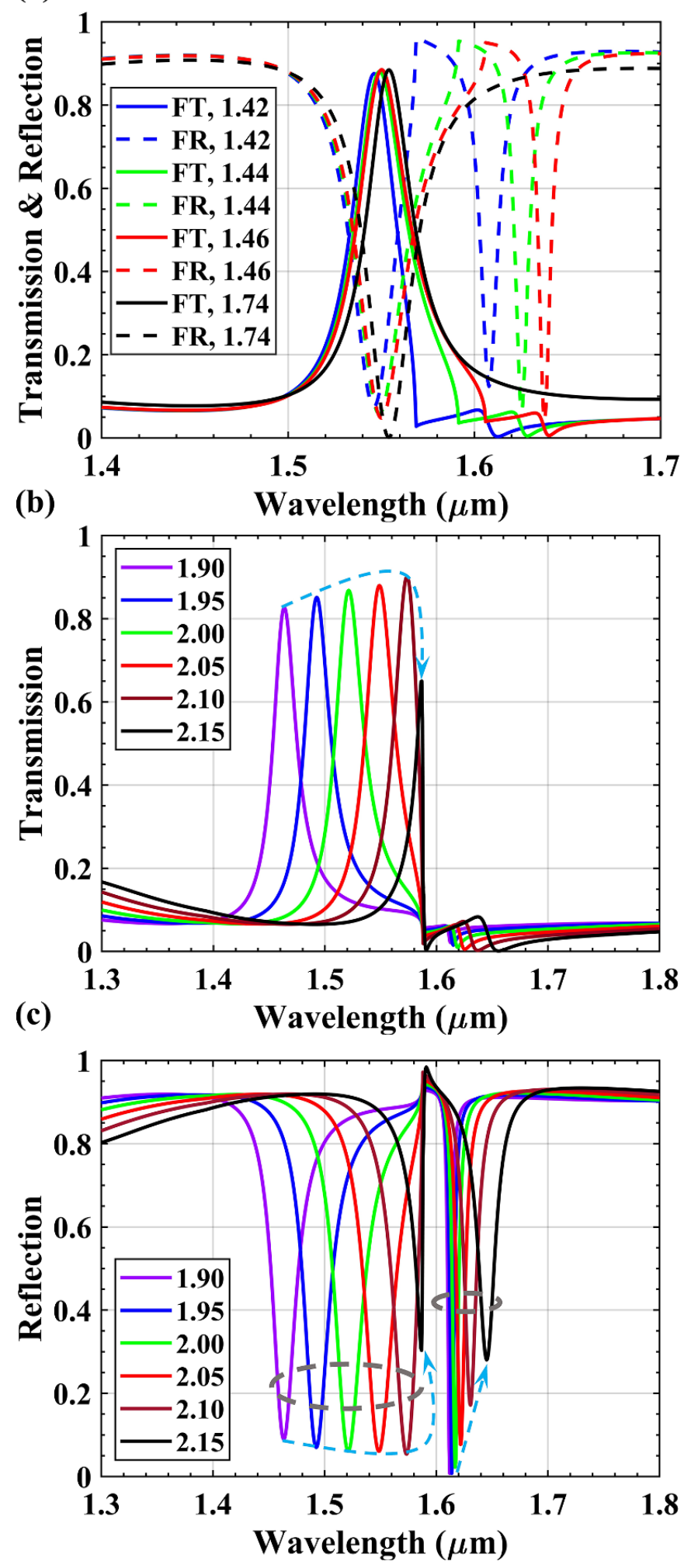

Figure 2. (a) Forward transmission/reflection (FT/FR) spectrum of the proposed structure (given in figure 1(a)) obtained for different substrates and corresponding refractive indices $\left(\mathrm{CaF}_{2}(\sim 1.42)<\mathrm{SiO}_{2}(\sim 1.44)<\mathrm{BaF}_{2}(\sim 1.46)<\mathrm{Al}_{2} \mathrm{O}_{3}(\sim 1.74)\right)$. The structure is illuminated by an $x$-polarized uniform plane wave propagating along the $-z$ directions. (b) Calculated forward transmission spectrum and (c) forward reflection spectrum of the proposed structure obtained for different superstrates (different refractive indices from 1.9 to 2.15 . 
(a)

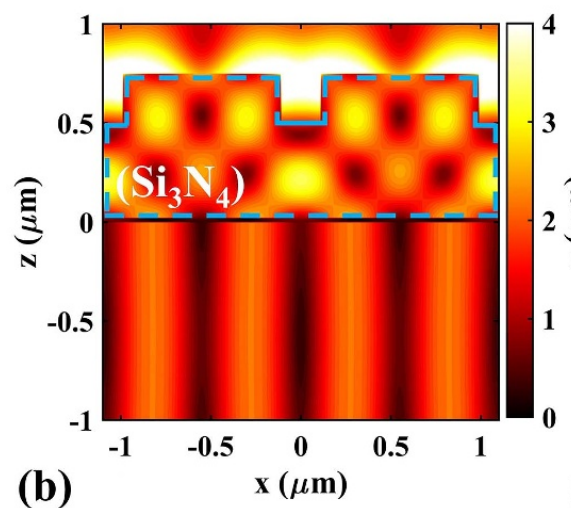

(b)

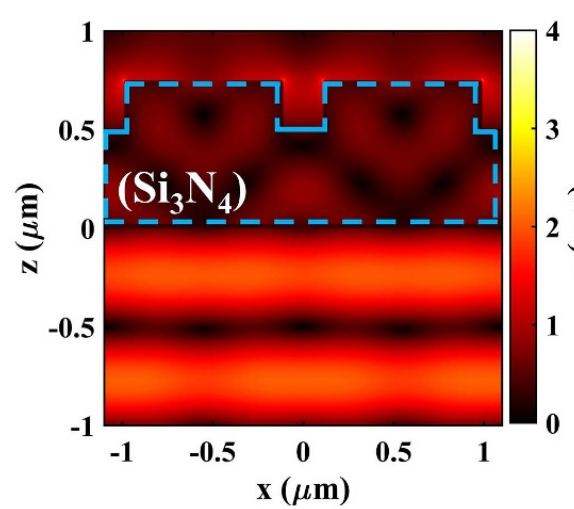

(c)

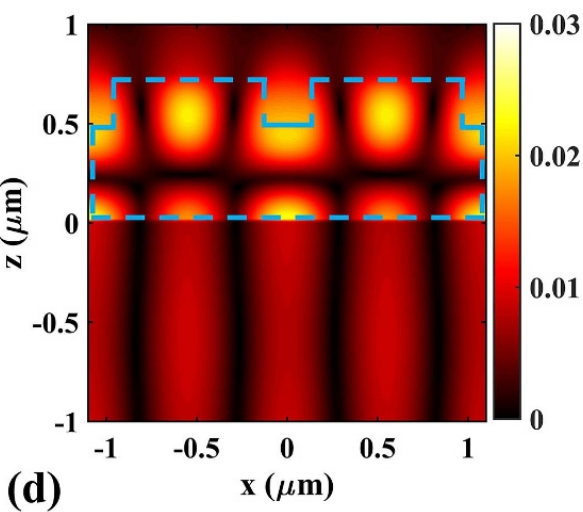

(e)
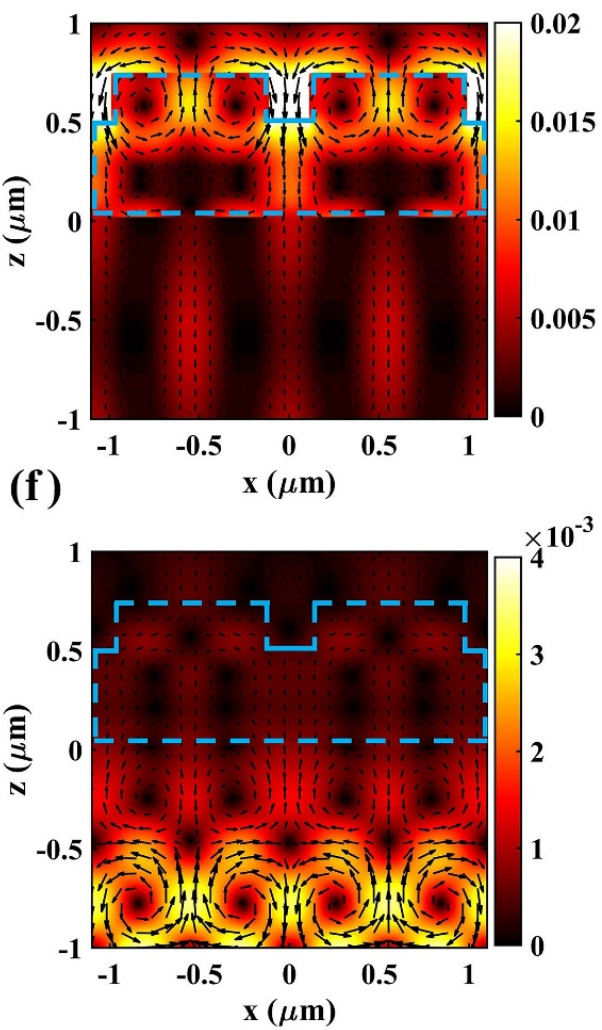

Figure 3. The absolute values of the total electric-field distributions on the $x-z$ plane obtained for a two-unit cell at the operating wavelength of $1549 \mathrm{~nm}$ (peak resonance of forward transmission spectrum) when the object is normally illuminated by an $x$-polarized uniform plane wave from the (a) front side (forward illumination) and (b) back side (backward illumination). The absolute values of the total magnetic-field distributions when the structure experiences (c) forward and (d) backward illuminations at the same operating wavelength. The Poynting-vector distributions when the object experiences (e) forward and (f) backward illuminations. In all figures, dashed blue lines represent the $\mathrm{Si}_{3} \mathrm{~N}_{4}$ area.

and LRSPP modes can be outcoupled to radiation modes [29], the analysis reveals that the small and large shifts corresponding to the first and second resonances are due to the SRSPP and LRSPP, respectively, and the SRSPP is tunneling and then dominantly outcoupled (induced) to the substrate, while LRSPP is the main reason for the absorption that appeared as a second resonance in the reflection spectrum. The same procedure continues for different superstrate materials and the results are separately given in figures 2(b) and (c) for the transmission and reflection spectra, respectively. It is seen that the 1 st resonance experiences larger red shifts than the 2nd resonance for different superstrate materials. This validates that the types of the 1st and 2nd resonances are due to the excitations of SRSPP and LRSPP, respectively.

\subsection{Near-field light-matter interactions at the resonance wavelength of $1549 \mathrm{~nm}$}

Near-field light-matter interactions of the proposed structure including on-resonance absolute values of electric- and magnetic-field distributions on the $x-z$ planes are shown in figures 3(a)-(d) for both-illumination sides. The electric field is partially confined in the waveguide layer for the forward illumination and it is mainly distributed in the groove medium as presented in figure 3(a). It is also seen that the guidedmode condition in the dielectric grating waveguide is satisfied, which led to the waveguide-coupled SPP excitation, and then the outcoupling of SPPs to the radiation modes. Moreover, as depicted in figure 3(c), the magnetic field is mainly confined within the grating structure which also shows the formation of the Fabry-Pérot cavity and the excitation of SPPs for the forward illumination [43, 44]. Therefore, the overall resonance of the designed structure for the forward illumination is due to the pairing of photonic (Fabry-Pérot) and plasmonic (SRSPP) modes. Under backward incidence, the absolute values of electric- and magnetic-field distributions at the operating wavelength of $1549 \mathrm{~nm}$ represent the formation of standing waves (partial reflection) in the substrate as shown in figures 3(b) and (d), respectively. The low value of backward transmission within the operating wavelengths is due to the fact that SPPs cannot be excited under backward incidence. In other words, the proposed diode-like device is operating based on the exciting and outcoupling of SPPs to radiation modes using an optimally designed dielectric-based diffraction grating waveguide for the forward illumination. While the backward incident electromagnetic wave cannot be coupled to the SPPs since there is no configuration that is identical to the one in the front side to excite the SPPs [45]. Thereby, the 
(a)
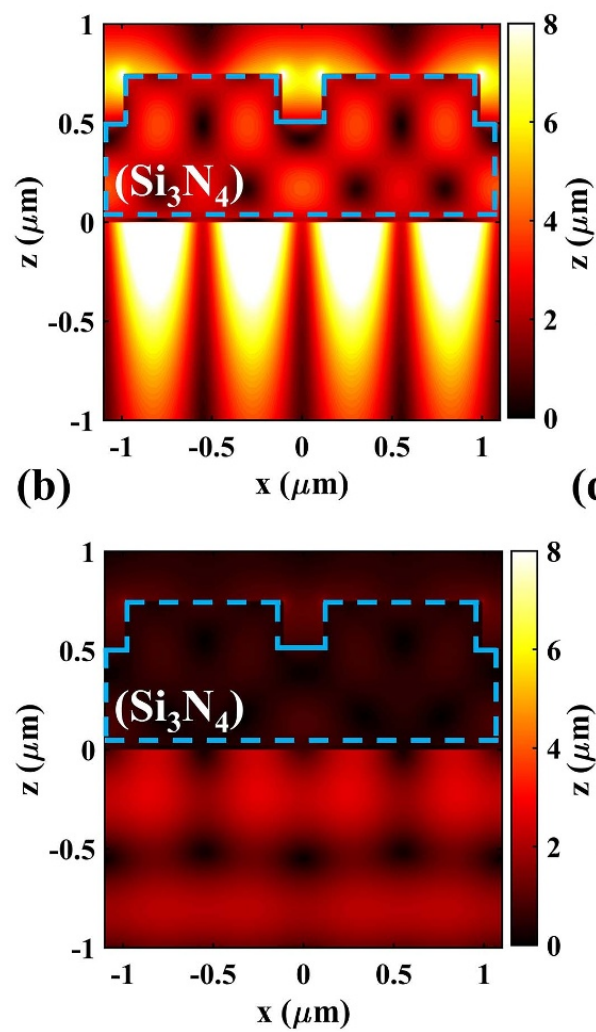

(c)

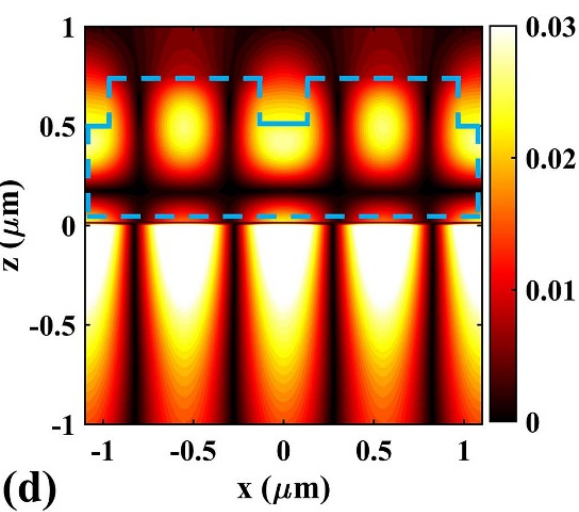

(e)

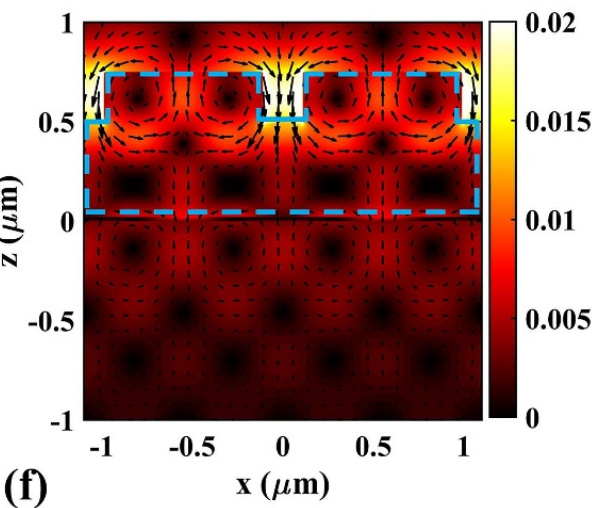

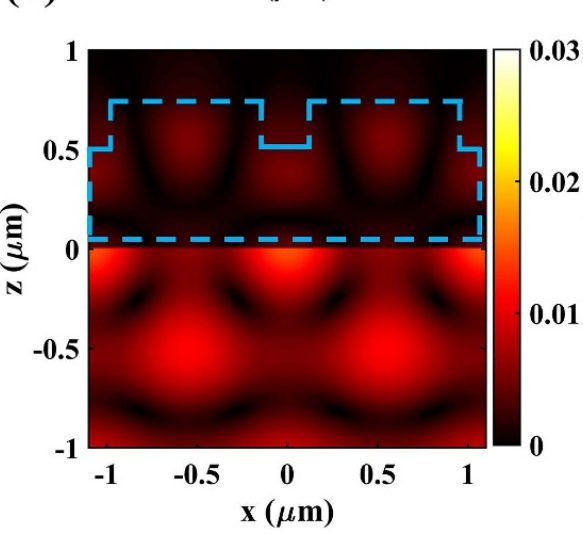

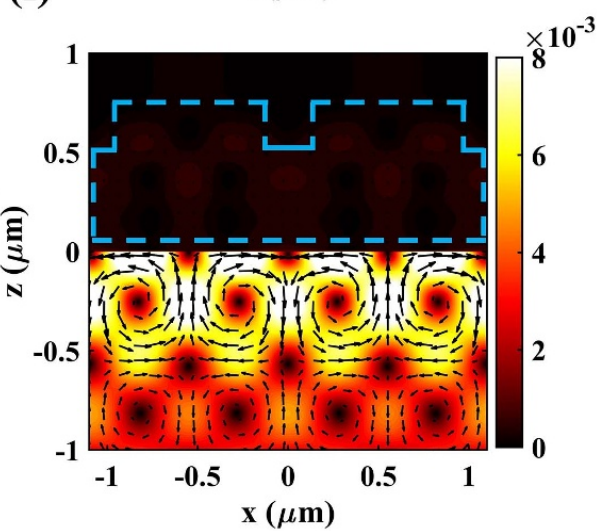

Figure 4. The absolute values of the total electric-field distributions on the $x-z$ plane obtained for a two-unit cell at the operating wavelength of $1624 \mathrm{~nm}$ (2nd dip resonance of forward reflection spectrum) when the object is normally illuminated by an $x$-polarized uniform plane wave from the (a) front side (forward illumination) and (b) back side (backward illumination). The absolute values of the total magnetic-field distributions when the structure experiences (c) forward and (d) backward illuminations at the same operating wavelength. The Poynting-vector distributions when the object experiences (e) forward and (f) backward illuminations. In all figures, dashed blue lines represent the $\mathrm{Si}_{3} \mathrm{~N}_{4}$ area.

structure offers strong reflection by the Ag film under backward incidence and the incident wave mostly reflects back. This results in the reciprocity breaking and obtaining a highcontrast AT response in the proposed geometry. In addition to the computed absolute values of electric and magnetic fields at the resonance wavelength of $1549 \mathrm{~nm}$, the Poynting-vector distributions, which reflect the average power flow directions, are presented for the forward and backward illuminations in figures 3(e) and (f), respectively. The results demonstrate that the considerable amount of average power can reach the next environment for the forward illumination in contrast to the blockage of the power in the reverse direction.

\subsection{Near-field light-matter interactions at the resonance wavelength of $1624 \mathrm{~nm}$}

Near-field light-matter interaction results, including absolute values of electric- and magnetic-field distributions for both forward and backward illuminations, are shown in figure 4(a) through figure $4(d)$ for a better visualization of the type of the second dip resonance $(1624 \mathrm{~nm}$, pertaining to the reflection spectrum of the forward illumination). The excitation of LRSPPs under the forward illumination at the bottom interface of the Ag layer is readily observed from the electric-field and magnetic-field distributions as seen in figures 4(a) and (c), respectively. The Poynting-vector distribution, shown in figure 4(e) for the forward illumination at the resonance wavelength of $1624 \mathrm{~nm}$, confirms that LRSPPs do not contribute to the radiation in the next environment and are the main reason for the absorption in the metallic layer. In a similar fashion to the operating wavelength of $1549 \mathrm{~nm}$, the structure offers strong reflection by the Ag film under backward incidence as shown in figures 4(b), (d), and (f), corresponding to the electric-field, magnetic-field, and Poynting-vector distributions, respectively. This is due to the fact that the configuration cannot support the excitation of SPPs and the plasmon tunneling from the back side.

\subsection{Absorbed power density}

For further investigation, the absolute values of absorbed power densities on the $x-z$ plane and in the vicinity of the Ag layer for both-illumination sides are considered at the resonance wavelengths of the reflection spectrum and shown in figure 5. The absorbed power density obtained at the resonance wavelength of $1549 \mathrm{~nm}$ for the forward illumination is 
(a)

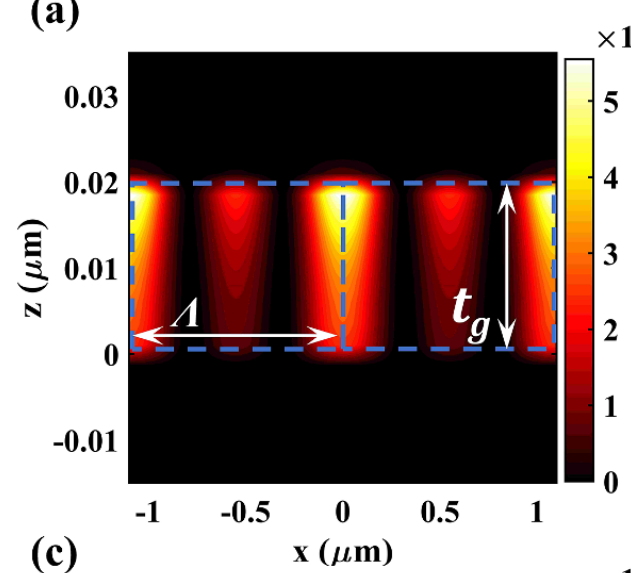

(c)

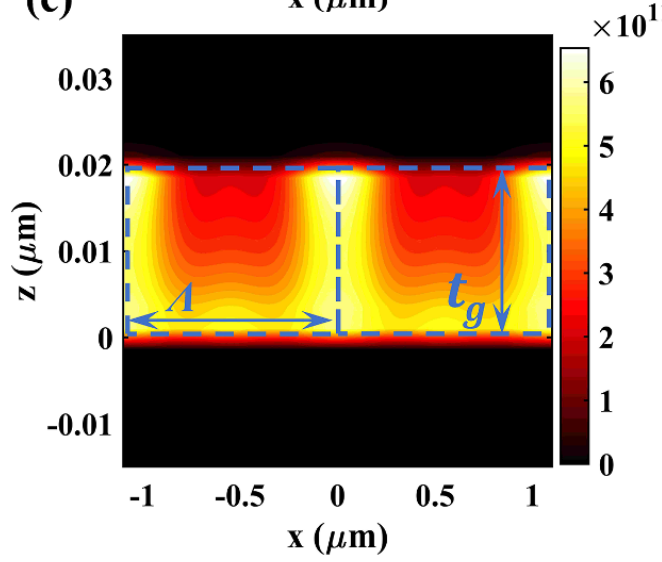

(b)
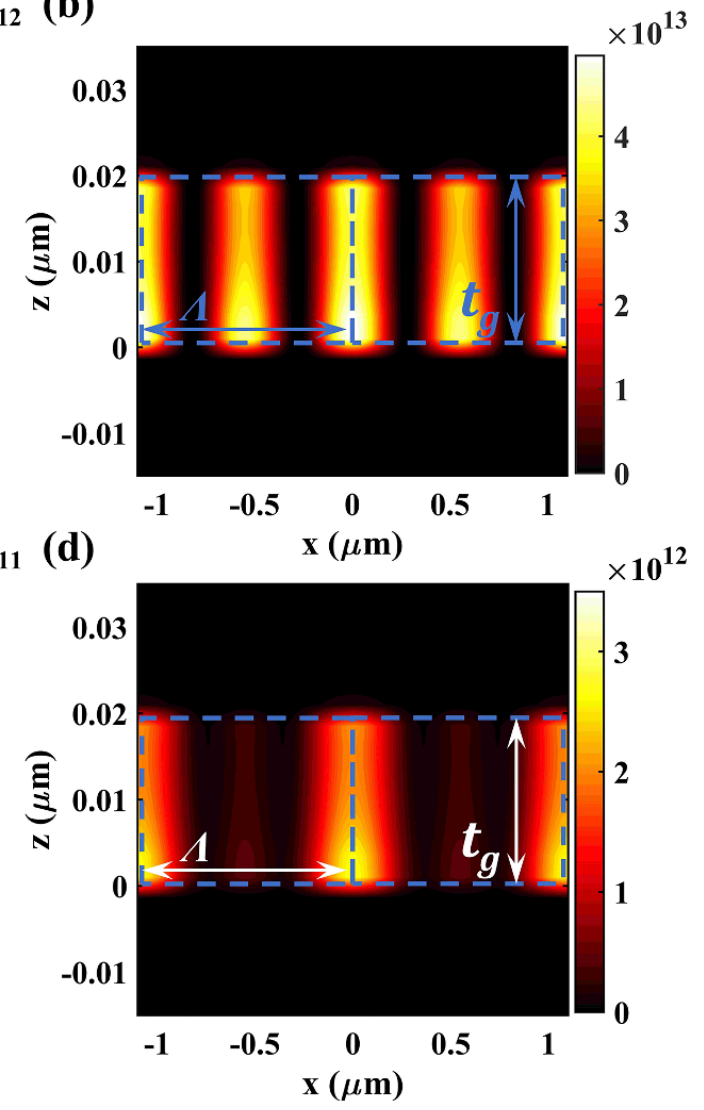

Figure 5. Absolute values of absorbed power densities obtained for a two-unit cell at the resonance wavelengths of (a) 1549 nm and (b) $1624 \mathrm{~nm}$ when the proposed AT structure is illuminated by an $x$-polarized uniform plane wave from the front side. Absolute values of absorbed power densities obtained for a two-unit cell at the resonance wavelengths of (c) $1549 \mathrm{~nm}$ and (d) $1624 \mathrm{~nm}$ when the proposed AT structure is illuminated by an $x$-polarized uniform plane wave from the back side. In all figures, dashed blue lines represent the Ag area.

given in figure 5(a). It is seen that the transparency of the structure is due to the SPs excited on the top interface of the Ag layer. These SPs are attributed to the dispersion relation given in (4a), so the dominant excitation mode is SRSPP as demonstrated using different superstrate materials (see figure 2) and the field distributions (see figure 3). Regarding the absorbed power density at the second resonance $(1624 \mathrm{~nm})$, as shown in figure 5(b), the SPs excited in the bottom layer are the dominant ones and cause the second reflection dip in the reflection spectrum under forward incidence. Therefore, the great part of the absorption of the structure is due to the SPs excited on the bottom interface of the silver layer. These SPs are related to the dispersion given in (4b), so the excitation mode is LRSPP as demonstrated using different substrate materials (see figure 2) and the field distributions (see figure 4). According to figures 5(c) and (d) for the backward illumination at the operating wavelengths of 1549 and $1624 \mathrm{~nm}$, respectively, it is observed that small amounts of the electromagnetic wave can be absorbed within the Ag layer due to the penetration of the electromagnetic wave, while these quantities are considerably smaller in comparison to their counterparts obtained from the forward illumination, demonstrating that most of the incident electromagnetic wave reflects.

\subsection{Geometric parameters effects}

It is also possible to provide an efficient optical-diode behavior in the desired operating wavelengths by using different values of the geometric parameters. As a result, the impact of different geometries on the resonance features of each of these modes have been studied as shown in figures 6 and 7. Under forward incidence, the SRSPPs excited by the dielectric diffraction grating at the upper interface can tunnel through the Ag film, when the thickness of the metallic layer is smaller than the penetration depth of the SRSPPs [21, 33, 34]. Therefore, the thickness of the Ag layer plays an important role in the efficient tunneling of the SRSPPs based on their penetration depth [21]. The simulated forward and backward transmission power versus the wavelengths are presented in figures 6(a) and (b), respectively, for different thicknesses of the Ag layer $\left(t_{\mathrm{m}}: 5-60 \mathrm{~nm}\right)$ when the other dimensional parameters are being kept fixed at their initial (optimal) values. As expected, it is observed that the AT property of the proposed structure is strongly dependent on the thickness of the metallic layer. Under forward incidence (see figure 6(a)), it promotes the efficient tunneling of SPPs, and thereby helps to increase forward transmission. While under backward incidence (see 
(a)

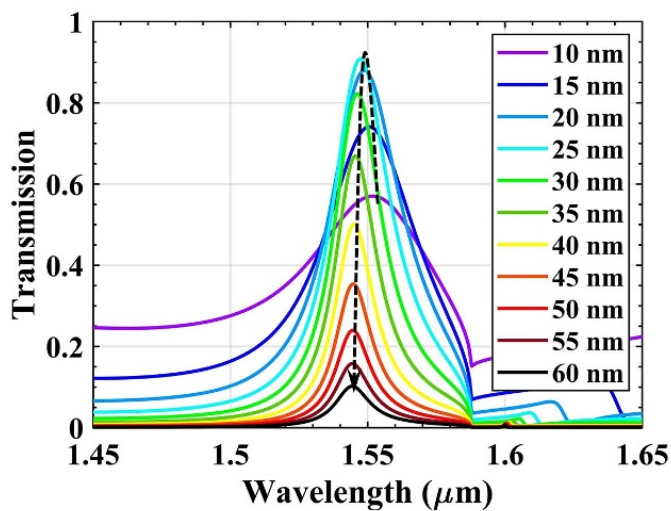

(c)

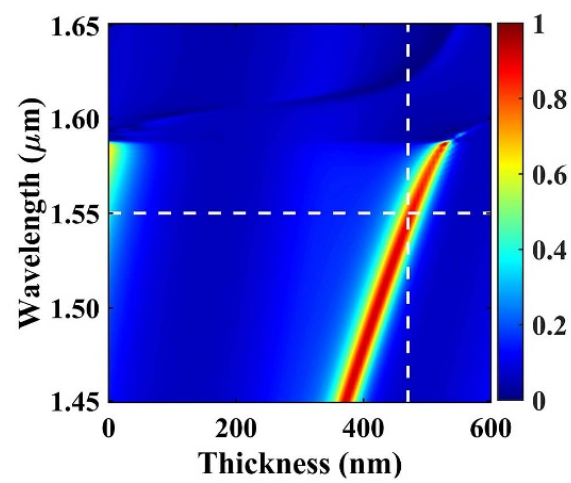

(b)

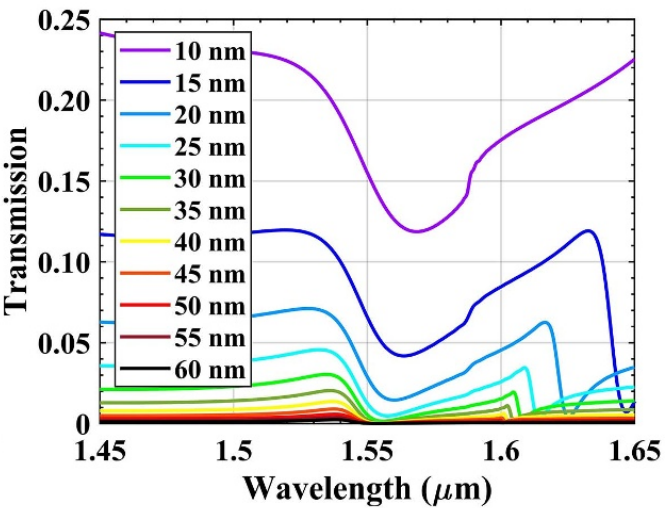

(d)

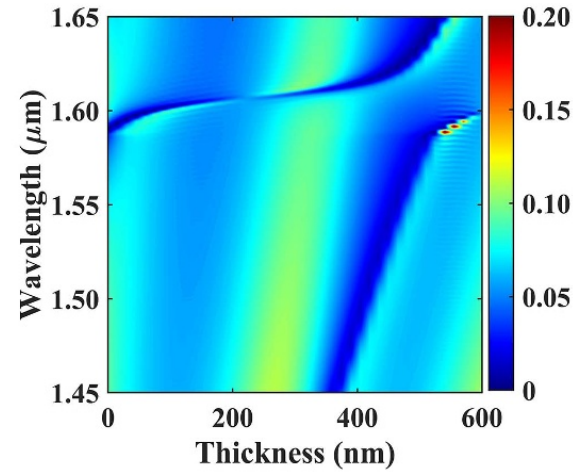

Figure 6. Dependency of the forward (1st column) and backward (2nd column) transmission spectra of the proposed optical diode on the variation of geometry parameters including: (a), (b) the thickness of the metal layer $\left(10 \mathrm{~nm}<t_{\mathrm{m}} \leq 60 \mathrm{~nm}\right.$ when $t_{\mathrm{g}}=260 \mathrm{~nm}$, $w_{\mathrm{g}}=880 \mathrm{~nm}, t_{\mathrm{d}}=470 \mathrm{~nm}$ and $\left.\Lambda=1100 \mathrm{~nm}\right)$, (c), (d) the thickness of the dielectric slab $\left(0<t_{\mathrm{d}}<600 \mathrm{~nm}\right.$ when $t_{\mathrm{g}}=260 \mathrm{~nm}$, $w_{\mathrm{g}}=880 \mathrm{~nm}, t_{\mathrm{m}}=20 \mathrm{~nm}$ and $\Lambda=1100 \mathrm{~nm}$ ). The interaction point of the white dashed lines corresponds to the operating wavelength of $1549 \mathrm{~nm}$.

figure 6(b)), it enhances reflection and thereby reduces transmission. Therefore, it is critical to choose suitable thickness for the Ag film. The thick Ag layer mitigates mixed mode SPP excitations and leads to unidirectional transmission blockage. However, at the optimal values of between 20 and $25 \mathrm{~nm}$, the design has high on-resonance forward transmission with a near zero off-resonance backward response. On the other hand, when the Ag film is thinner $\left(t_{\mathrm{m}}=15\right.$ and $\left.10 \mathrm{~nm}\right)$, the strong near-field coupling will change the excitation condition of SPPs, and then accordingly change the corresponding SPPs wavelength, resulting in a decrease in the forward transmittance at the operating wavelength.

Another prominent geometry parameter is the thickness of the slab waveguide. This parameter can be utilized to tune the SPP resonances and improve the overall performance of the proposed structure as seen in figures 6(c) and (d) representing forward and backward transmissions, respectively. Using this geometry, the resonance wavelength and its strength can be deliberately engineered. As seen in these figures, without this tuner slab, the resonance property (amplitude and FWHM) of the design is quite poor. The SPPs can be efficiently excited when the phase of the diffracted waves in the dielectric slab matches the phase of the surface plasmon waves in the silver film. Therefore, this layer adds a degree of freedom to provide a better matching condition and stronger resonance response.
The coupling efficiency of the proposed structure is also strongly dependent on the periodicity, height, and width of the grating as a fact that the normal incident wave first interacts with the grating and the grating determines the property of the diffracted waves. Therefore, the changes in the grating size seriously affect the performance of the structure and the excitation of SPPs. Figure 7 illustrates the effects of the grating width and the grating height with respect to the wavelengths for both forward transmission and forward reflection cases. It is clear that, as the dimensions of the grating increase, at the specific width and height of the grating, the guided-mode resonance and the phase-matched conditions are satisfied, and the AT property is achieved. In addition, the spectral positions of the SPP modes vary and experience a red-shifted phenomenon in a linear fashion due to the occurring the FabryPérot cavity modes and then corresponding SPP modes at longer wavelengths (provides phase-matching condition for another wavelength). As seen in figures 7(a) and (b), the most important part is that SPP cannot be excited when the width of grating structure is equal to 1.1 (the filling factor is 1 ), while increasing the height of the grating part decreases the effects of diffracted waves involved in the excitation of the SPPs as seen in figures 7(c) and (d). All in all, the first resonance is affected more by changing the grating dimensions due to the occurring SRSPP between the Ag layer and the waveguide slab 
(a)

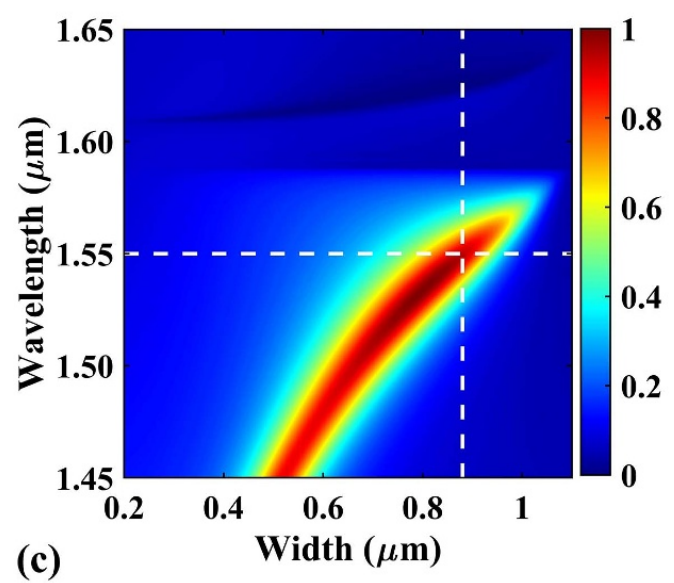

(c)

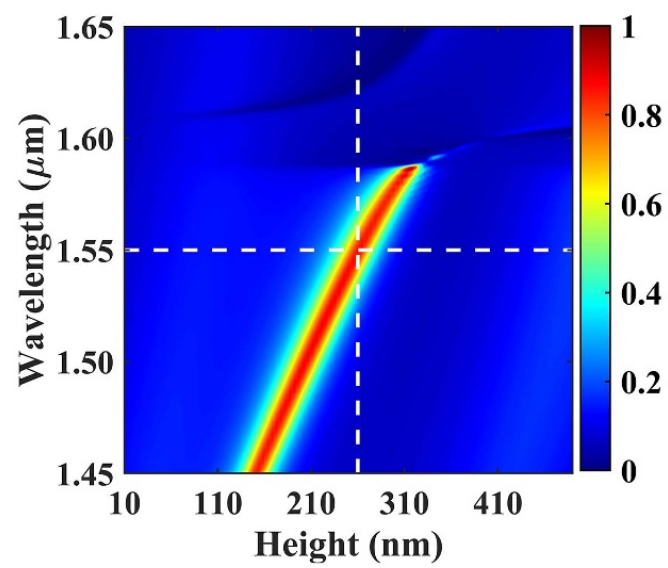

(b)
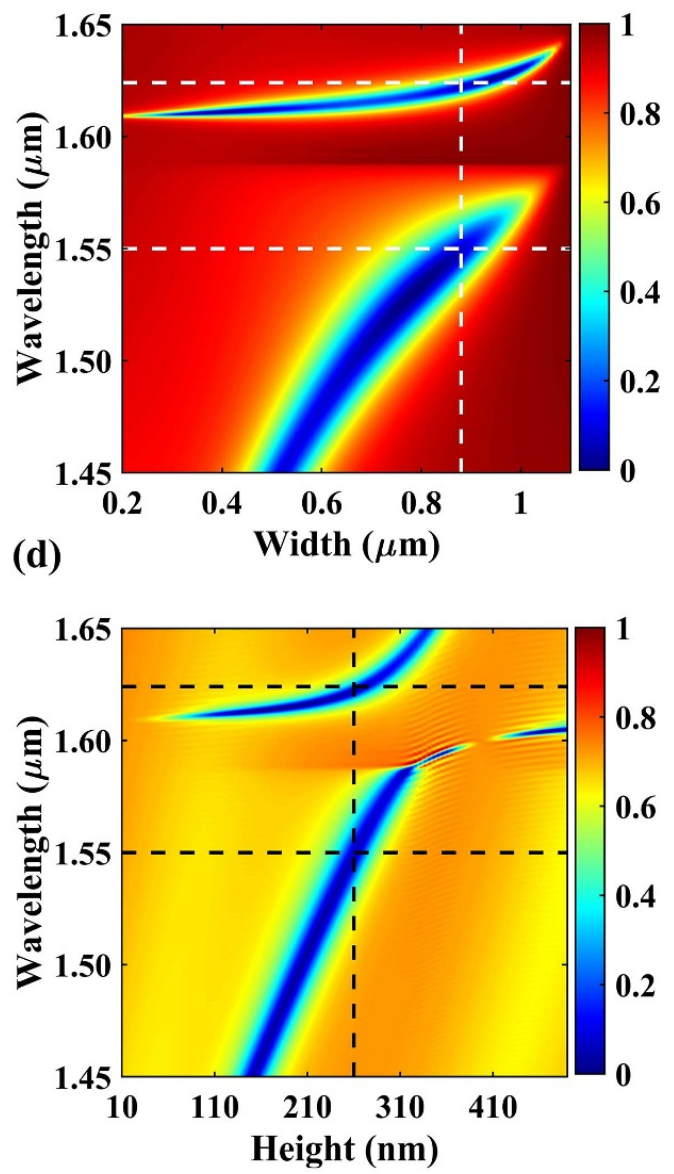

Figure 7. Dependency of the normalized transmission (1st column) and reflection (2nd column) spectra of the proposed structure on the variation of geometry parameters including: (a), (b) the width of grating $\left(200 \mathrm{~nm} \leq w_{\mathrm{g}} \leq 1100 \mathrm{~nm}\right.$ when $t_{\mathrm{g}}=260 \mathrm{~nm}, t_{\mathrm{m}}=20 \mathrm{~nm}$, $t_{\mathrm{d}}=470 \mathrm{~nm}$ and $\left.\Lambda=1100 \mathrm{~nm}\right)$, and (c), (d) the height (thickness) of the grating $\left(10 \mathrm{~nm} \leq t_{\mathrm{g}} \leq 490 \mathrm{~nm}\right.$ when $t_{\mathrm{d}}=470 \mathrm{~nm}, w_{\mathrm{g}}=880 \mathrm{~nm}$, $t_{\mathrm{m}}=20 \mathrm{~nm}$ and $\Lambda=1100 \mathrm{~nm}$ ). The interaction points of the dashed lines correspond to the operating wavelengths of 1549 and $1624 \mathrm{~nm}$.

in comparison to the second resonance (LRSPP) occurs at the bottom layer.

\section{Conclusions}

In conclusion, we proposed a simple periodic nano array design with AT property which can be considered as an optical diode. The proposed device is made up of a grating waveguide on a thin metal layer coated on a substrate, and it works based on the excitations of SPPs at both interfaces of the thin metal layer and plasmon-tunneling effect. The plasmontunneling effect is based on the transmission provided by the SRSPP excited at the upper interface. In contrast to the other works, the proposed design has a high-contrast asymmetric light transmission ratio in the NIR and provide nearzero inverse transmission. The blocking is caused by the metal layer where the SPP cannot be excited. The spectral behaviors of the proposed design are analyzed and validated by using RCWA besides the FDTD method in order to prove the proposed concept. Furthermore, the emerging nanofabrication techniques will allow the fabrication of our design where the operating principle is not limited to the NIR region, and it can be extended to a different range of the spectrum due to the scalable property and flexible design of the proposed structure.

\section{Data availability statement}

All data that support the findings of this study are included within the article (and any supplementary files).

\section{Acknowledgment}

One of the authors (E O) acknowledges partial support from the Turkish Academy of Sciences.

\section{Conflict of interest}

The authors declare no conflict of interest. 


\section{ORCID iDs}

Bahram Khalichi (D) https://orcid.org/0000-0002-9465-1044 Ataollah Kalantari Osgouei (D) https://orcid.org/0000-00020971-7687

\section{References}

[1] Li Z, Mutlu M and Ozbay E 2013 Chiral metamaterials: from optical activity and negative refractive index to asymmetric transmission J. Opt. 15023001

[2] Shi J H, Ma H F, Guan C Y, Wang Z P and Cui T J 2014 Broadband chirality and asymmetric transmission in ultrathin 90-twisted babinet-inverted metasurfaces Phys. Rev. B 89165128

[3] Davis T J, Eftekhari F, Gómez D E and Roberts A 2019 Metasurfaces with asymmetric optical transfer functions for optical signal processing Phys. Rev. Lett. 123013901

[4] Li Z, Mutlu M and Ozbay E 2014 Highly asymmetric transmission of linearly polarized waves realized with a multilayered structure including chiral metamaterials J. Phys. D: Appl. Phys. 47075107

[5] Kuzmiak V and Maradudin A A 2012 Asymmetric transmission of surface plasmon polaritons Phys. Rev. A 86043805

[6] Singh R, Plum E, Menzel C, Rockstuhl C, Azad A K, Cheville R A and Zheludev N I 2009 Terahertz metamaterial with asymmetric transmission Phys. Rev. B 80153104

[7] Kuzmiak V and Maradudin A A 2015 Asymmetric transmission of surface plasmon polaritons on planar gratings Phys. Rev. A 92053813

[8] Xu P, Lv X, Chen J, Li Y, Qian J, Chen Z and Xu J 2018 Dichroic optical diode transmission in two dislocated parallel metallic gratings Nanoscale Res. Lett. 13 1-8

[9] Mutlu M, Akosman A E, Serebryannikov A E and Ozbay E 2012 Diodelike asymmetric transmission of linearly polarized waves using magnetoelectric coupling and electromagnetic wave tunneling Phys. Rev. Lett. 108213905

[10] Huang C, Feng Y, Zhao J, Wang Z and Jiang T 2012 Asymmetric electromagnetic wave transmission of linear polarization via polarization conversion through chiral metamaterial structures Phys. Rev. B 85 1-5

[11] Ismail Khan M, Hu B, Chen Y, Ullah N, Khan M J I and Khalid A R 2020 Multiband efficient asymmetric transmission with polarization conversion using chiral metasurface IEEE Antennas Wirel. Propag. Lett. $191137-41$

[12] Menzel C, Helgert C, Rockstuhl C, Kley E B, Tünnermann A, Pertsch T and Lederer F 2010 Asymmetric transmission of linearly polarized light at optical metamaterials Phys. Rev. Lett. $1041-4$

[13] Kang M, Chen J, Cui H-X, Li Y and Wang H-T 2011 Asymmetric transmission for linearly polarized electromagnetic radiation Opt. Express 198347

[14] Shang X J, Zhai X, Wang L L, He M D, Li Q, Luo X and Duan H G 2017 Asymmetric transmission and polarization conversion of linearly polarized waves with bilayer L-shaped metasurfaces Appl. Phys. Express 10052602

[15] Liu D J, Xiao Z Y, Ma X L and Wang Z H 2015 Asymmetric transmission of linearly and circularly polarized waves in metamaterial due to symmetry-breaking Appl. Phys. Express 8052001

[16] Li Z, Liu W, Cheng H, Chen S and Tian J 2016 Tunable dual-band asymmetric transmission for circularly polarized waves with graphene planar chiral metasurfaces Opt. Lett. $413142-5$
[17] Li X, Feng R and Ding W 2018 Extremely high contrast asymmetric transmission with linear tunability in chiral metamaterials J. Phys. D: Appl. Phys. 51145304

[18] Pan C, Ren M, Li Q, Fan S and Xu J 2014 Broadband asymmetric transmission of optical waves from spiral plasmonic metamaterials Appl. Phys. Lett. 104121112

[19] Li Y, Dong G, Zhao R, Wang K, Zhou S, Sun L and Shi J 2018 Dual-band asymmetric transmission and circular dichroism in hybrid coupled plasmonic metamaterials J. Phys. D: Appl. Phys. $\mathbf{5 1} 285105$

[20] Ling Y, Huang L, Hong W, Liu T, Sun Y, Luan J and Yuan G 2017 Asymmetric optical transmission based on unidirectional excitation of surface plasmon polaritons in gradient metasurface Opt. Express 25 13648-58

[21] Ba C, Huang L, Liu W, Li S, Ling Y and Li H 2019 Narrow-band and high-contrast asymmetric transmission based on metal-metal-metal asymmetric gratings $O p t$. Express 27 25107-18

[22] Pfeiffer C, Zhang C, Ray V, Guo L J and Grbic A 2014 High performance bianisotropic metasurfaces: asymmetric transmission of light Phys. Rev. Lett. 113023902

[23] Cakmakyapan S, Serebryannikov A E, Caglayan H and Ozbay E 2010 One-way transmission through the subwavelength slit in nonsymmetric metallic gratings $O p t$. Lett. 35 2597-9

[24] Homola J, Yee S S and Myszka D 2008 Surface plasmon resonance biosensors Optical Biosensors 185-242

[25] Abutoama M and Abdulhalim I 2015 Self-referenced biosensor based on thin dielectric grating combined with thin metal film Opt. Express 23 28667-82

[26] Sharma A K and Pandey A K 2019 Self-referenced plasmonic sensor with $\mathrm{TiO}_{2}$ grating on thin Au layer: simulated performance analysis in optical communication band J. Opt. Soc. Am. B 36 F25-F31

[27] Park S, Lee G, Song S H, Oh C H and Kim P S 2003 Resonant coupling of surface plasmons to radiation modes by use of dielectric gratings Opt. Lett. 28 1870-2

[28] Lenaerts C, Michel F, Tilkens B, Lion Y and Renotte Y 2005 High transmission efficiency for surface plasmon resonance by use of a dielectric grating Appl. Opt. 44 6017-22

[29] Shen S, Forsberg E, Han Z and He S 2007 Strong resonant coupling of surface plasmon polaritons to radiation modes through a thin metal slab with dielectric gratings $J$. Opt. Soc. Am. A 24 225-30

[30] Wedge S and Barnes W L 2004 Surface plasmon-polariton mediated light emission through thin metal films $O p t$ Express 12 3673-85

[31] Byun K M, Kim S J and Kim D 2007 Grating-coupled transmission-type surface plasmon resonance sensors based on dielectric and metallic gratings Appl. Opt. 46 5703-8

[32] Hu C and Liu D 2009 Transmission-type SPR sensor based on coupling of surface plasmons to radiation modes using a dielectric grating Front. Optoelectron. China 2 182-6

[33] Zhang L, Hao J, Ye H, Yeo S P, Qiu M, Zouhdi S and Qiu C W 2013 Theoretical realization of robust broadband transparency in ultrathin seamless nanostructures by dual blackbodies for near infrared light Nanoscale 5 3373-9

[34] Zhou L, Wen W, Chan C T and Sheng P 2005 Electromagnetic-wave tunneling through negative-permittivity media with high magnetic fields Phys. Rev. Lett. 94 1-4

[35] Xu T and Lezec H J 2014 Visible-frequency asymmetric transmission devices incorporating a hyperbolic metamaterial Nat. Commun. 5 1-7

[36] Zinkiewicz Ł, Haberko J and Wasylczyk P 2015 Highly asymmetric near infrared light transmission in an all-dielectric grating-on-mirror photonic structure $\mathrm{Opt}$. Express 23 4206-11 
[37] Zeng L, Chen M, Yan W, Li Z and Yang F 2020 Si-grating-assisted SPR sensor with high figure of merit based on Fabry-Pérot cavity Opt. Commun. 457124641

[38] Johnson P B and Christy R W 1972 Optical constants of the noble metals Phys. Rev. B 64370

[39] Balanis C A 2012 Advanced Engineering Electromagnetics (New York: Wiley)

[40] Han Z and Bozhevolnyi S I 2012 Radiation guiding with surface plasmon polaritons Rep. Prog. Phys. 76016402

[41] Dionne J A, Sweatlock L A, Atwater H A and Polman A 2005 Planar metal plasmon waveguides: frequency-dependent dispersion, propagation, localization, and loss beyond the free electron model Phys. Rev. B 72075405
[42] Moharam M G, Grann E B, Pommet D A and Gaylord T K 1995 Formulation for stable and efficient implementation of the rigorous coupled-wave analysis of binary gratings J. Opt. Soc. Am. A 12 1068-76

[43] Buhara E, Ghobadi A, Khalichi B, Kocer H and Ozbay E 2021 Mid-infrared adaptive thermal camouflage using a phase-change material coupled dielectric nanoantenna J. Appl. Phys. 54265105

[44] Kalantari Osgouei A, Hajian H, Khalichi B,

Serebryannikov A E, Ghobadi A and Ozbay E 2021 Active tuning from narrowband to broadband absorbers using a sub-wavelength $\mathrm{VO}_{2}$ embedded layer Plasmonics $1-9$

[45] Maier S A 2007 Plasmonics: Fundamentals and Applications (New York: Springer Science and Business Media) 